\title{
Analysis of Sheepdog-Type Robot Navigation for Goal-Lost-Situation
}

\author{
Yuichiro Sueoka *, Makihiko Ishitani and Koichi Osuka \\ Department of Mechanical Engineering, Graduate School of Engineering, Osaka University, 2-1 Yamadaoka, \\ Suita, Osaka 565-0871, Japan; m.ishitani@dsc.mech.eng.osaka-u.ac.jp (M.I.); \\ osuka@mech.eng.osaka-u.ac.jp (K.O.) \\ * Correspondence: sueoka@mech.eng.osaka-u.ac.jp; Tel.: +81-6-6879-4084
}

Received: 16 April 2018; Accepted: 17 May 2018; Published: 18 May 2018

\begin{abstract}
In the real world, there is a system in which a dog called a sheepdog stimulates part of a flock of sheep that are freely moving to guide them to a goal position. If we consider this system from the perspective of a control problem, it is an interesting control system: one or more sheepdogs, who act as a small number of controllers, are used to indirectly control many sheep that cannot be directly controlled. For this reason, there have been many studies conducted regarding this system; however, these studies have been limited to building numerical models or performing simulation analyses. Very little research has been done on building a working system. The point we wish to emphasise here is that we attempted to build the sheepdog system in as simple a way as possible. For the purpose, we introduce minimal settings for the sheep model and the sheepdog controller. In the process of building and testing an actual system, we noticed "an emergence of blind zone" because the robots possess size, or so-called cases where the objects in the blind zone cannot be observed because the object is in front. Using the existing method, as the number of sheep increases, it becomes impossible to perceive the goal position, i.e., emerge the goal-lost-situation. This results in the guidance task becoming impossible. As clear identification of the goal position is vital for guidance, we propose a method for cases in which the goal position is invisible. Using our method, the robot appropriately selects another object, and sets this object as the new target. We have confirmed through simulations that the proposed method can maintain guidance regardless of the number of sheep.
\end{abstract}

Keywords: distributed autonomous system; sheepdog-type navigation; local information

\section{Introduction}

On grazing land, there is a system in which a flock of several thousands of sheep that run about freely are controlled by only a few dogs called sheepdogs. Sheep instinctively gather together in flocks and flee from sheepdogs, and a sheepdog guides sheep by skillfully taking advantage of these characteristics. Considering this system from the perspective of a control problem, it forms an extremely interesting control system in which sheepdogs, as a small number of dynamic controllers, are used to indirectly control a flock of sheep that they cannot control directly. In our research, we call this "sheepdog-type navigation". We believe that if it could be realized as a robot system it would be socially significant, thus we set out to create such a system.

There are some related works concerned with animal navigation other than the sheepdog system. For example, Correll [1] has tried to manipulate the cattle by examining the effect of the animal states, such as stress. Butler [2] has examined the fence or fence line design which will affect the group behavior based on the knowledge of the cattle. Additional previous research on zebras ([3]) and insects $[4,5]$ explained challenges monitoring states of farm animals or insects. To achieve the purpose, 
these works adopt an approach to integrate sensory information such as whole position/velocity information. For robotic application, navigation techniques also rely on GPS sensors and complicated network design ([6,7]).

Prior research on sheepdog systems was firstly conducted by modeling the sheep and the sheepdogs and reproducing the guiding behavior of the sheepdog [8]. Other research has proposed and verified many sheep and sheepdog models from the perspectives of degree of similarity to behavior of an actual sheepdog system, and guidance performance in guiding a flock of sheep [9-14]. In [10], the sheep were modeled in relatively high detail. In [11,12], guidance was proposed by having a sheepdog move to the left and right behind the sheep to prevent them from scattering. In [13], a method for preventing a flock of sheep from being split up (by implementing an action to bring the sheep together when they have scattered) was proposed. Lastly, in [14], a comparative verification of multiple control methods for sheepdogs was performed.

Many behavioral algorithms for the sheepdogs have been proposed in this literature and were determined to be appropriate. Conversely, we have noticed that past research did not consider the size of the objects modeled, but has treated the sheep and the sheepdogs as material points, meaning the models are not applicable to living animals and robots. When guiding a large group of robots, there will be cases of a robot being obscured by the robot in front of it, or cases in which the goal position is invisible because it is obscured by a robot or animal. As the viability of achieving guidance in such cases is unknown, this study seeks to determine recommendations for the guidance of many sheep robots by performing guidance experiments on a small number of sheep robots in order to achieve sheepdog-type robot navigation. The point we wish to emphasise here is that we intend to build the sheepdog system in as simple a way as possible. For this purpose, we examine the blind-zone effect in the experiment after introducing minimal settings for the sheep model and sheepdog algorithm from Couzin [15] and Vaughan [8].

The detailed research procedures are as follows. After building the model of sheep and sheepdog, we carried out fundamental experiments. In the experiment, the relative positional relationship of the robots is obtained by attaching LED and illuminance sensors to the sheep robots and the dog robot. In addition, a test of the guidance of three sheep robots by one dog robot was carried out. In the case where it was possible to perceive the light emitted by the other robot (a case with few blind zones) guidance was performed successfully, but we noticed cases where the dog robot could not perceive the sheep robot or the goal. Thus, it is assumed that in a case where the number of sheep is increased, the influence of blind zones would be greater, resulting in guidance failure. On the basis that the guidance impact of a dog robot is unable to perceive the goal results from the object having size rather than being modelled as a point, we proposed the following method: "Using another object that replaces the goal as a substitute goal".

1. When the goal is visible, the positional relationship of the position of the goal with the center of mass of the sheep (visible zone) is remembered.

2. When the goal has become invisible, the relative relationship of the position of the goal just before it became invisible with the direction of the center of the sheep is compared. If the position of the goal is to the left of the center of the sheep, the goal is the sheep on the leftmost side of the sheep that are visible. The same rule applies in the case where it is to the right.

To verify the appropriateness of the proposed method, a simulation model with blind zones (zones in which the goal is invisible) is constructed, and the guidance performance of the past method and of the proposed method are compared by varying the number of sheep and performing statistical analysis.

This paper is constructed as follows. First, in Section 2, the models of the sheep and sheepdog are explained, the two-wheeled robot made for the actual experiment is introduced. Section 3 demonstrates the specifics of the experiment using this robot in a case with three sheep. In Section 4 , the appropriateness of the proposed method is verified by a simulation considering blind zones. Finally, in Section 5, the paper is summed up. 


\section{Actual Experiment of Sheepdog-Type Robot Navigation}

The model discussed here uses a sheep robot (sheep) and a dog robot (sheepdog). For the sheep, a concentric circle model widely known as a model of a flock of animals [15] is used, and for the sheepdog, the center targeting control method proposed by past research [8] is used.

\subsection{Model of the Sheep}

This paper refers to the Boid model as the model of the sheep. A Boid model is a model in which, "flock behavior is achieved by localized activity decisions which individuals make in response to the state of their own surroundings", and it can represent flock behavior based on the following three principles [16]:

- Separation: separates from individuals within a separation zone to avoid contact

- Alignment: adapts to the direction and speed of individuals in the nearby zone

- Cohesion: approaches the center direction of other individuals in the nearby zone

Additionally, Couzin et al. proposed the concentric circle model depicted in Figure 1, showing the forces received in response to these three interactions according to the distance between individuals. In the concentric circle model in Figure 1, in the zone where the distance $r$ between individuals is $r<r_{s}$, repulsion, in the zone where $r_{s}<r<r_{a}$, alignment, and in the zone where $r_{a}<r<r_{c}$, cohesion is received $\left(r, r_{s}, r_{a}, r_{c} \in \mathbb{R}_{+}\right)$.

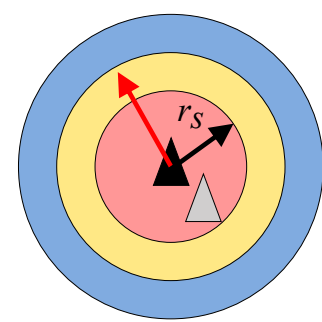

(a)

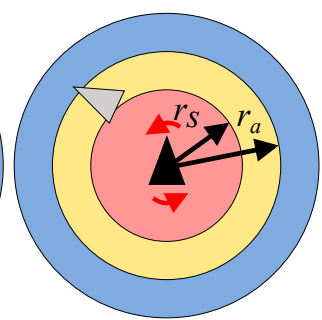

(b)

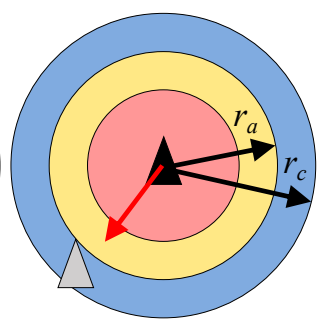

(c)

Figure 1. Concentric Circle model $\left(r \leq r_{s}\right.$ : separation radius; $r_{s}<r \leq r_{a}$ : alignment radius; $r_{a}<r \leq r_{c}$ : cohesion radius); (a) Separation; (b) Alignment; (c) Cohesion.

Additionally, repulsion $u_{i, 1}[k]$, alignment $u_{i, 2}[k]$, and cohesion $u_{i, 3}[k]$ that the $i(i=1 ; 2 ; \cdots ; n)$ th sheep receives at step $k \in \mathbb{N}_{0}$ respectively, are as shown below $\left(u_{i, 1}[k], u_{i, 2}[k], u_{i, 3}[k] \in \mathbb{R}^{2}\right)$.

$$
\begin{gathered}
u_{i, 1}[k]=-\sum_{j \in N_{i, s}} \frac{x_{j}[k]-x_{i}[k]}{\left|x_{j}[k]-x_{i}[k]\right|} \\
u_{i, 2}[k]=\sum_{j \in N_{i, a}} \frac{u_{j}[k]-u_{i}[k]}{\left|x_{j}[k]-x_{i}[k]\right|} \\
u_{i, 3}[k]=\sum_{j \in N_{i, c}} \frac{x_{j}[k]-x_{i}[k]}{\left|x_{j}[k]-x_{i}[k]\right|} \\
N_{i, s}=\left\{j \in\{1,2, \cdots, n\} \backslash\{i\}|| x_{j}[k]-x_{i}[k] \mid<r_{s}\right\} \\
N_{i, a}=\left\{j \in\{1,2, \cdots, n\} \backslash\{i\}\left|r_{s}<\right| x_{j}[k]-x_{i}[k] \mid<r_{a}\right\} \\
N_{i, c}=\left\{j \in\{1,2, \cdots, n\} \backslash\{i\}\left|r_{a}<\right| x_{j}[k]-x_{i}[k] \mid<r_{c}\right\}
\end{gathered}
$$

Here, $N_{i, s}, N_{i, a}$ and $N_{i, c}$ are the subset of sheep group. $x_{i}[k]$ and $u_{i}[k] \in \mathbb{R}^{2}$ represent the position and the speed respectively of the $i$ th sheep at step $k$, and $n \in \mathbb{N}_{+}$represents the number of sheep. 
The repulsion $u_{i, 4}[k] \in \mathbb{R}^{2}$ of the sheep escaping from the sheepdog is set as follows:

$$
u_{i, 4}[k]=-\frac{x_{d}[k]-x_{i}[k]}{\left|x_{d}[k]-x_{i}[k]\right|^{3}}
$$

where, $x_{d}[k] \in \mathbb{R}^{2}$ is the position of the sheepdog at step $k$. The $i$ th sheep receives the force of the combination of these four forces, and the speed $u_{i}[k]$ is calculated as shown below.

$$
u_{i}[k]=K_{s_{1}} u_{i, 1}[k]+K_{s_{2}} u_{i, 2}[k]+K_{s_{3}} u_{i, 3}[k]+K_{s_{4}} u_{i, 4}[k]
$$

where $K_{s_{1}}, K_{s_{2}}, K_{s_{3}}$, and $K_{s_{4}} \in \mathbb{R}_{+}$are gains. Position $x_{i}[k+1]$ of the sheep is calculated by the following formula based on speed $u_{i}[k]$.

$$
x_{i}[k+1]=x_{i}[k]+u_{i}[k]
$$

\subsection{Model of the Sheepdog}

The center targeting control method [8] proposed by past research is adopted for controlling the behavior of the sheepdog. This is a method for targeting using the center $G$ of a flock of sheep as the target as shown in Figure 2; its effectiveness is shown.

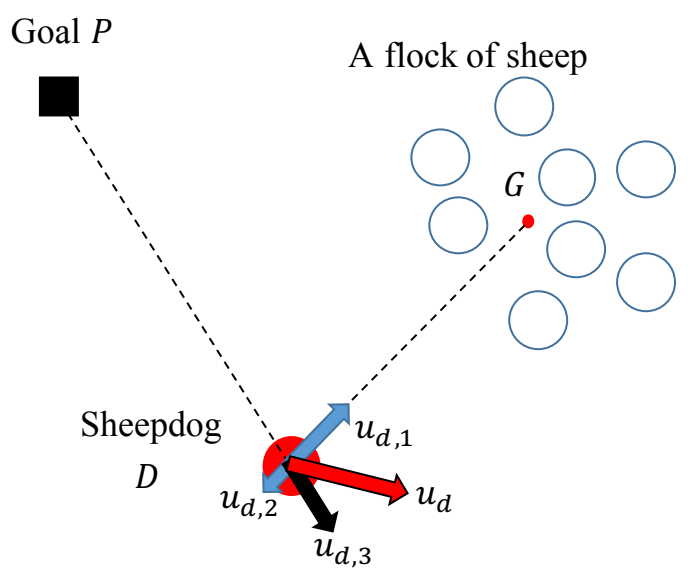

Figure 2. Center-targeting control: a sheepdog pursues the center of sheep.

Sheepdog $D$ received cohesion $u_{d, 1}[k] \in \mathbb{R}^{2}$ of a specified size to approach the center of the sheep and repulsion $u_{d, 2}[k] \in \mathbb{R}^{2}$ proportional to the square of the distance from the sheepdog to the center of the sheep to avoid approaching too close to the center of the sheep. Under the influence of these two forces, the sheepdog maintains an appropriate distance from the center of the flock. Additionally, it receives repulsion $u_{d, 3}[k] \in \mathbb{R}^{2}$ of a specified scale from goal $P$, and it moves around the flock of sheep to the opposite side of the goal. The sheepdog's speed $u_{d}[k] \in \mathbb{R}^{2}$ is represented by the following formulae.

$$
\begin{gathered}
u_{d, 1}[k]=\frac{x_{g}[k]-x_{d}[k]}{\left|x_{g}[k]-x_{d}[k]\right|} \\
u_{d, 2}[k]=-\frac{x_{g}[k]-x_{d}[k]}{\left|x_{g}[k]-x_{d}[k]\right|^{3}} \\
u_{d, 3}[k]=-\frac{x_{p}-x_{d}[k]}{\left|x_{p}-x_{d}[k]\right|} \\
u_{d}[k]=K_{d_{1}} u_{d, 1}[k]+K_{d_{2}} u_{d, 2}[k]+K_{d_{3}} u_{d, 3}[k]
\end{gathered}
$$


Here, $K_{d_{1}}, K_{d_{2}}, K_{d_{3}} \in \mathbb{R}_{+}$are gains, and $x_{g}[k]$ and $x_{p} \in \mathbb{R}^{2}$ represents the position of the center of the sheep and the position of the goal respectively. The position of the sheepdog $x_{d}[k+1]$ is calculated by the following formula based on the speed $u_{d}[k]$.

$$
x_{d}[k+1]=x_{d}[k]+u_{d}[k]
$$

\section{Achievement of a Sheepdog System Based on a Robot Group}

In this section, the modeled sheep and sheepdog are realized as a robot system. Figure 3a is an external view of the robots used in the study, 3pi robots sold on the market (made by Pololu), $10 \mathrm{~cm}$ in diameter, and equipped as follows:

- $\quad$ Microcomputer (mbed, made by ARM)

- $\quad$ IMU (made by BNO055, Bosch Sensortec)

- Communication module (XBee ZB(S2C), made by Digi International)

- High brightness LED (OS4WME56F1A, made by OptoSupply)

- Iluminance sensor (AMS302, made by Panasonic)

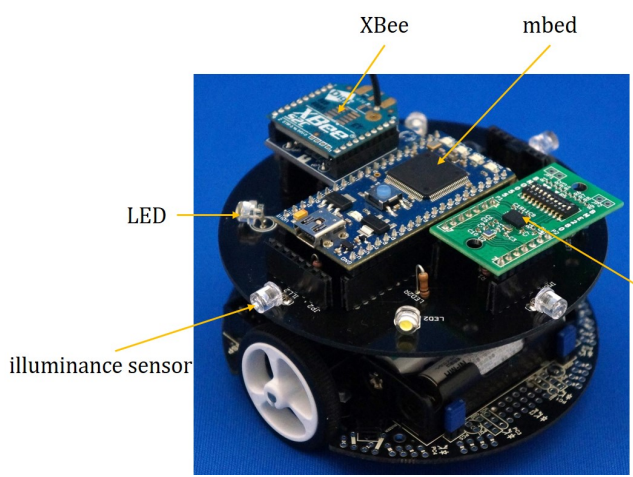

(a)

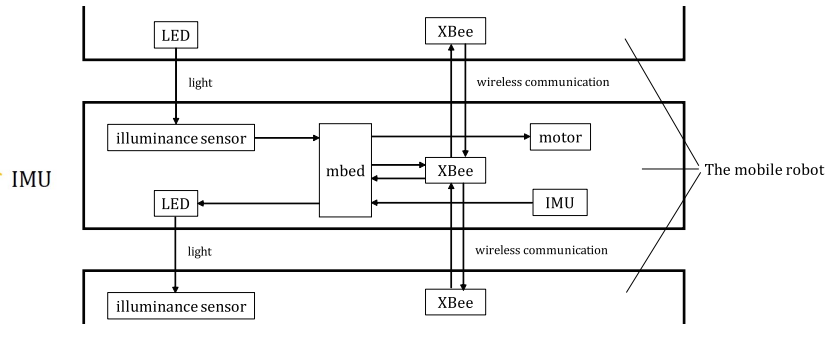

(b)

Figure 3. Developed robot system. (a) Overview of the mobile robot; (b) The block diagram of the mobile robots.

Figure $3 \mathrm{~b}$ shows a block wiring diagram for the robots. Each robot is equipped with four LED and four illuminance sensors to estimate relative position according to the intensity of the light emitted by the other robots. As shown in Figure 4, the illuminance sensors are installed so they are directed outwards at 90[deg] from the robot and from one another. The estimating robot obtains sensor value $I_{i} \in \mathbb{R}_{+}(i=1 ; \cdots ; 4)$ excluding offset from the four illuminance sensors. The vector is synthesized from the values obtained to calculate $I \in \mathbb{R}^{2}$. Specifically, as shown by formula (15), the direction is the unit vector $e_{i} \in \mathbb{R}^{2}$ in the direction of each illuminance sensor, and its scale sums up the vectors of $I_{i}$. The value of $I$ that is calculated, as shown in formula (16), is calculated such that the position $r \in \mathbb{R}^{2}$ of the robot that is estimated is at a distance inversely proportional to $\sqrt{|I|}$ in the direction of $I$. For example, in Figure 4, it is $I_{2}, I_{3}=0, I_{1}<I_{4}$, and the direction of the robot is judged to be $I=I_{1} e_{1}+I_{4} e_{4}$.

$$
\begin{gathered}
I=\sum_{i=1}^{4} I_{i} e_{i} \\
r=\frac{I}{|I|} \sqrt{\frac{k}{|I|}}
\end{gathered}
$$

However, $k \in \mathbb{R}_{+}$is a constant, and it is hypothesized that $|I|=k /|r|^{2}$ is established. 


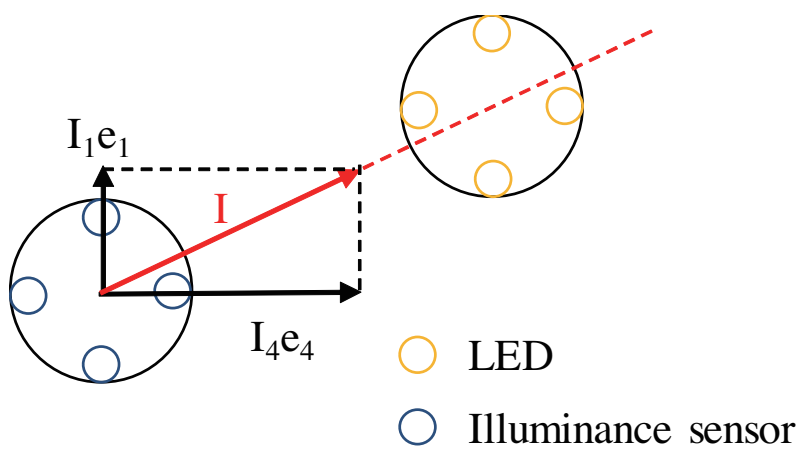

Figure 4. Decision of the relative position using LEDs and illuminance sensors.

To decide their direction of progress, each robot uses IMU. In order to obtain its own attitude, the sheep robot obtains the direction of other sheep robots and of the dog robot from values obtained by illuminance sensors, and the attitude of the other sheep robots obtained through using Xbee. A dog robot decides its direction of progress by obtaining the direction of the goal and of the sheep robots from the values from illuminance sensors. A sheep robot and a dog robot both control the direction of movement by PD control.

The actual experiment was done using three sheep robots, one dog robot, and one goal. Each of the robots is identified by an integer from 1 to 5 (1: sheepdog, 2-4: sheep, 5: goal) and emits the light in numerical order. If both the sheep robots and dog robot could not obtain light from another robot, it is assumed that they did not receive force from that robot. Figure 5 shows photographs taken during the experiment. Figure 6 shows positional data for the robots, obtained by motion capture at the start and at the end of the experiment, and Figure 7 shows the tracks of the robots during the experiment. In Figure 7, the position of the dog robot is shown by the circle symbol, that of the sheep robots by the triangle symbol, and that of the goal by the asterisk symbol. Figures 5-7 confirm that the sheepdog was able to guide the flock of sheep to the goal. When guiding three sheep, the impact of blind zones (cases where a certain robot cannot obtain the light of another robot) during measurement of relative positions by light was small, so guidance could be achieved by ignoring this robot, but as the number of sheep robots increased, cases where the goal is invisible occurred with increasing frequency, leading us to conclude that their impact becomes impossible to ignore.

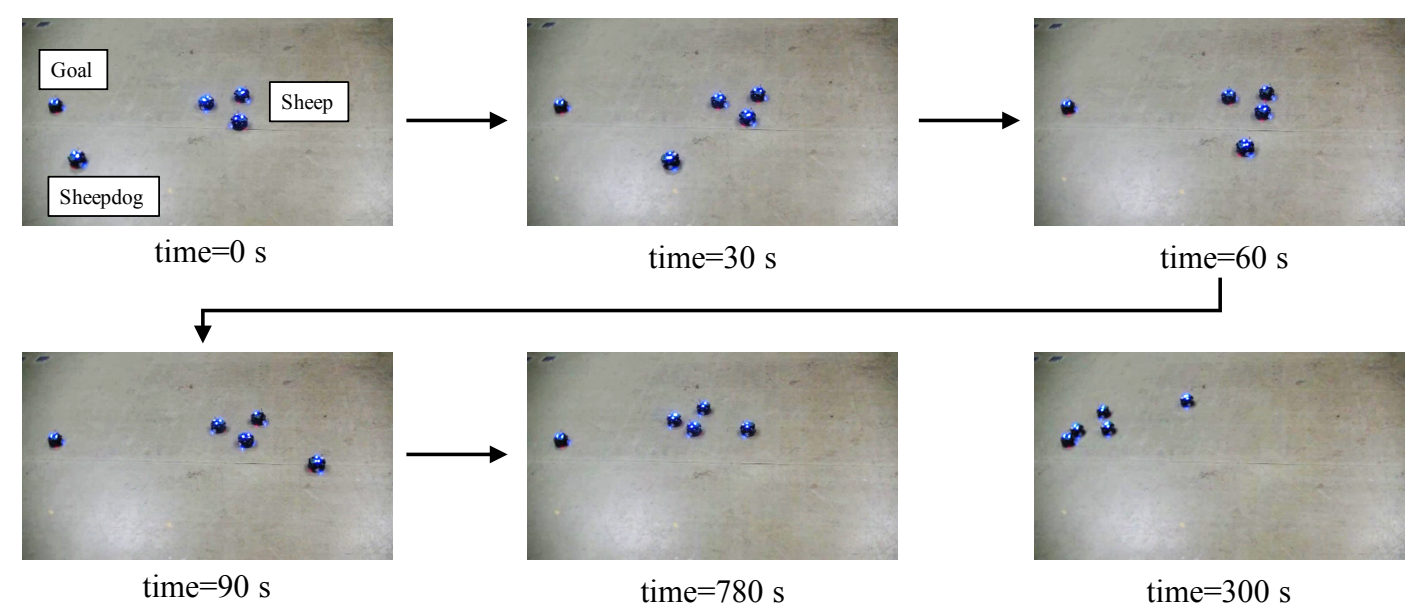

Figure 5. Snapshots of the guiding experiment. 


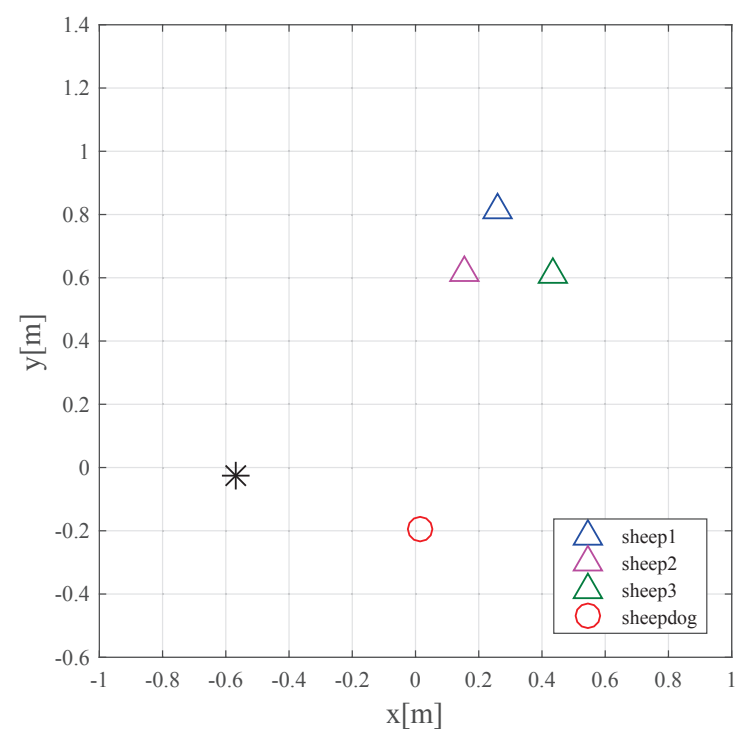

(a)

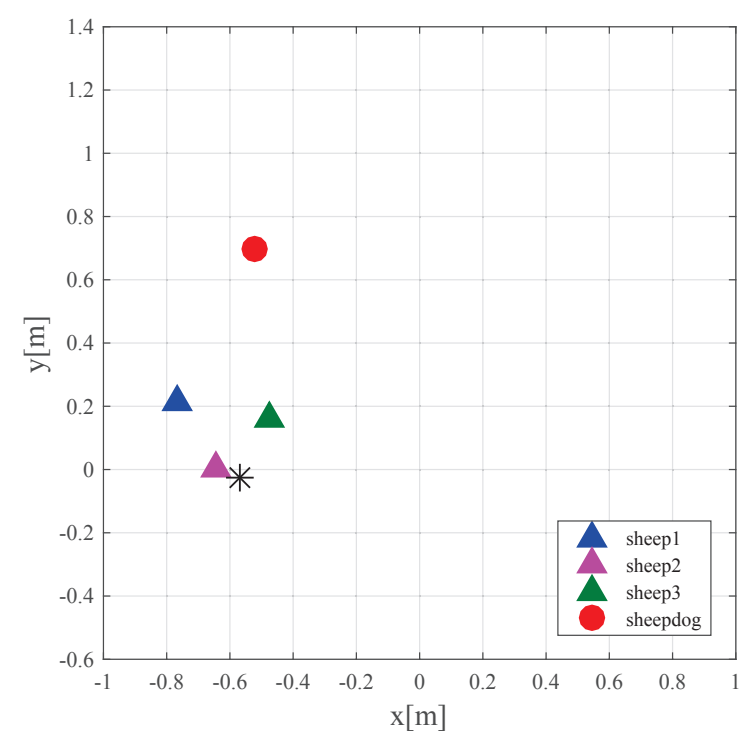

(b)

Figure 6. Initial states and final states. (a) Initial states; (b) Final states.

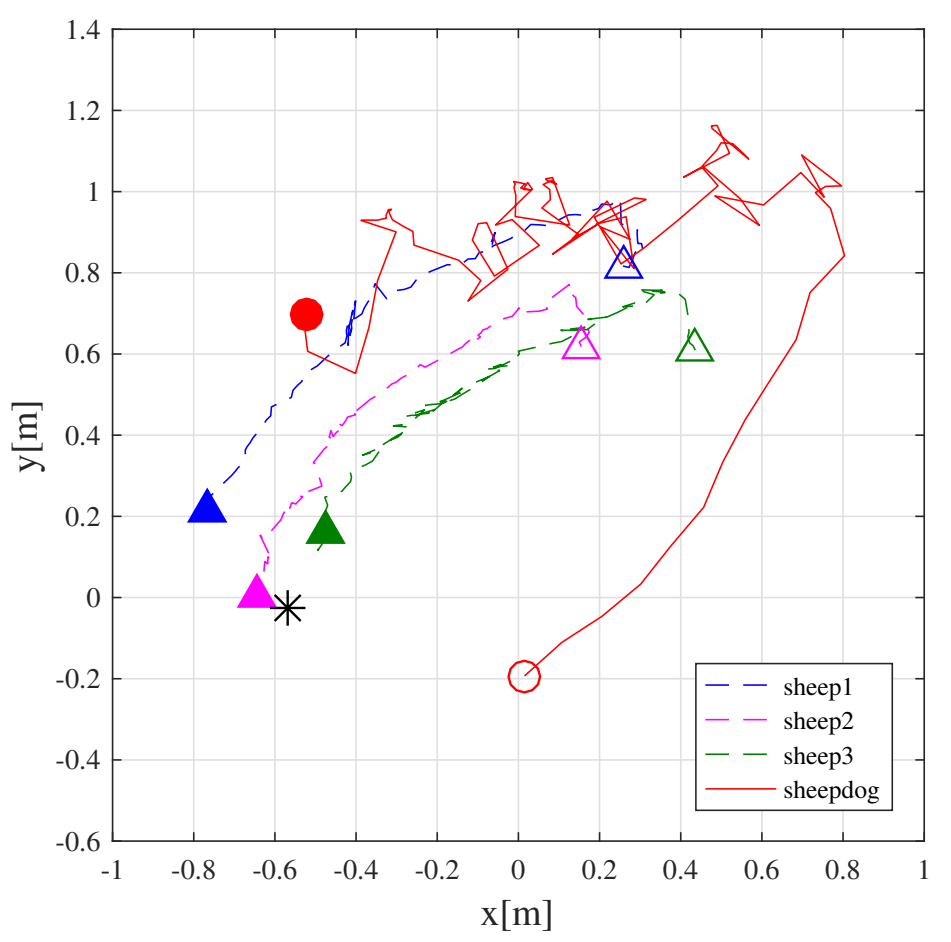

Figure 7. Trajectories of the sheep robots and that of the dog robot.

\section{Proposal and Analysis of a Guidance Method for Goal-Lost-Situation}

In this section, the issue of "losing sight of the position of the goal accompanying an increase in number of sheep" is experimentally investigated, and a method is proposed to realize guidance even when the sight of the goal is lost. First a simulation is built that considers the size of the robots. Later, analysis is done while varying the number of sheep, to learn how guidance performance is different in a case where the proposed method is combined with the center targeting control method, and in a case where it is not. 


\subsection{Simulation Settings}

Parameters for the sheep and sheepdog in the simulation are set as listed in Table 1. Parameters for which values are not set by later simulations use the values in Table 1. At the start of the simulation, sheep are placed randomly in a range $(0,0)-(800,800)$, and the initial positions of the sheepdog and of the goal are $(0,-800)$ and $(-400,-400)$ respectively. The speed of the sheep and of the sheepdog in the initial state is $(0,0)$. The simulation was fun up to 1500 steps.

Table 1. Parameters in the simulation.

\begin{tabular}{ccc}
\hline Parameter & Description & Value \\
\hline$n$ & Number of sheep & 30 \\
$K_{s_{1}}$ & Coefficient of separate force of sheep & 4 \\
$K_{s_{2}}$ & Coefficient of alignment force of sheep & 2 \\
$K_{s_{3}}$ & Coefficient of cohesion force of sheep & 2 \\
$K_{s_{4}}$ & Coefficient of repulsion force from sheepdog & 400,000 \\
$r_{s}$ & Radius of separation area & 70 \\
$r_{a}$ & Radius of alignment area & 490 \\
$r_{c}$ & Radius of cohesion area & 5000 \\
$K_{d_{1}}$ & Coefficient of attraction force from sheep & 30 \\
$K_{d_{2}}$ & Coefficient of repulsion force from sheep & $2,400,000$ \\
$K_{d_{3}}$ & Coefficient of repulsion force from goal & 12 \\
$R$ & Radius of an agent & 30 \\
\hline
\end{tabular}

\subsection{Definition of the Blind Zone Based on Size of the Agent}

This sub-section defines the "blind zone" in the simulation. As an example, the visible zone and blind zone of agent $i$ in Figure $8 \mathrm{a}$ are discussed. Seen from agent $i$, the blind zones created by the near agents overlap. Zone $A_{1}$ in Figure $8 \mathrm{a}$ is made a blind zone caused by agent $j$ that is the nearest. It is assumed that agent $i$ cannot perceive an agent with its entire body included in this zone, and that an agent that protrudes into a zone where it is partly visible can be perceived. Thus, the agent closest to the second from agent $i$ as shown in Figure $8 \mathrm{~b}$ is zone $A_{2}$ that is also a blind zone. Because zones $A_{1}$ and $A_{2}$ are both blind zones, agent $i$ cannot perceive an agent completely included in zones $A_{1} \cup A_{2}$. Similarly, considering zones $A_{3}, A_{4}$-which are made blind zones by the agent closest to the $3 \mathrm{rd}$, 4th-from Agent $i$, assuming that the number of agents is $N \in \mathbb{N}_{+}$, agent $i$ cannot perceive agents included completely in zones $A_{1} \cup A_{2} \cup A_{3} \cup A_{4} \cup \cdots \cup A_{n}$. In the simulation, the effect of blind zone is taken into account for all of the agents: the occlusion from sheep to sheep, that of sheep to sheepdog and vice versa.

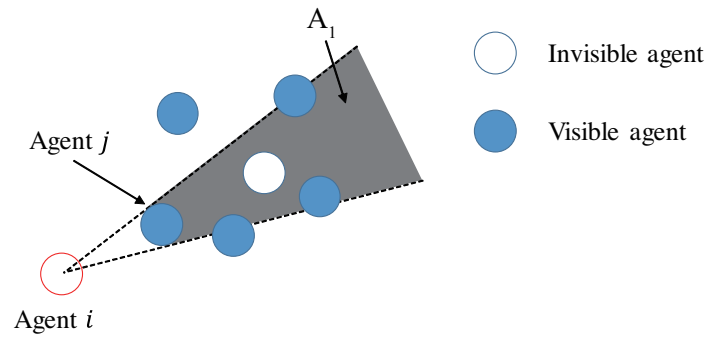

(a)

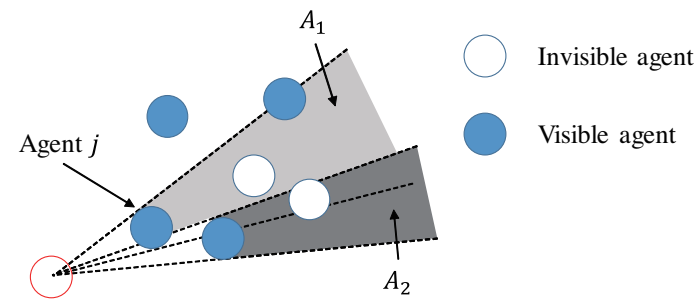

Agent $i$

(b)

Figure 8. Definition of the blind zone due to the agents' size. (a) Illustration of the blind zone made by the nearest agent; (b) Integration of each blind zone.

Figure 9a shows the results of guidance performed assuming all sheep are visible, and Figure $9 \mathrm{~b}$ shows the results when a blind zone appropriate to the size of the agent was introduced. Figure $9 \mathrm{a}$ 
confirms that in a case where there is no blind zone, guidance is possible, and Figure $9 \mathrm{~b}$ that incorporating consideration of a blind zone, smooth guidance is impossible. This is assumed to occur because in a case where there is a blind zone, during the experiment, the goal can no longer be perceived because of a flock of sheep, resulting in guidance failure.
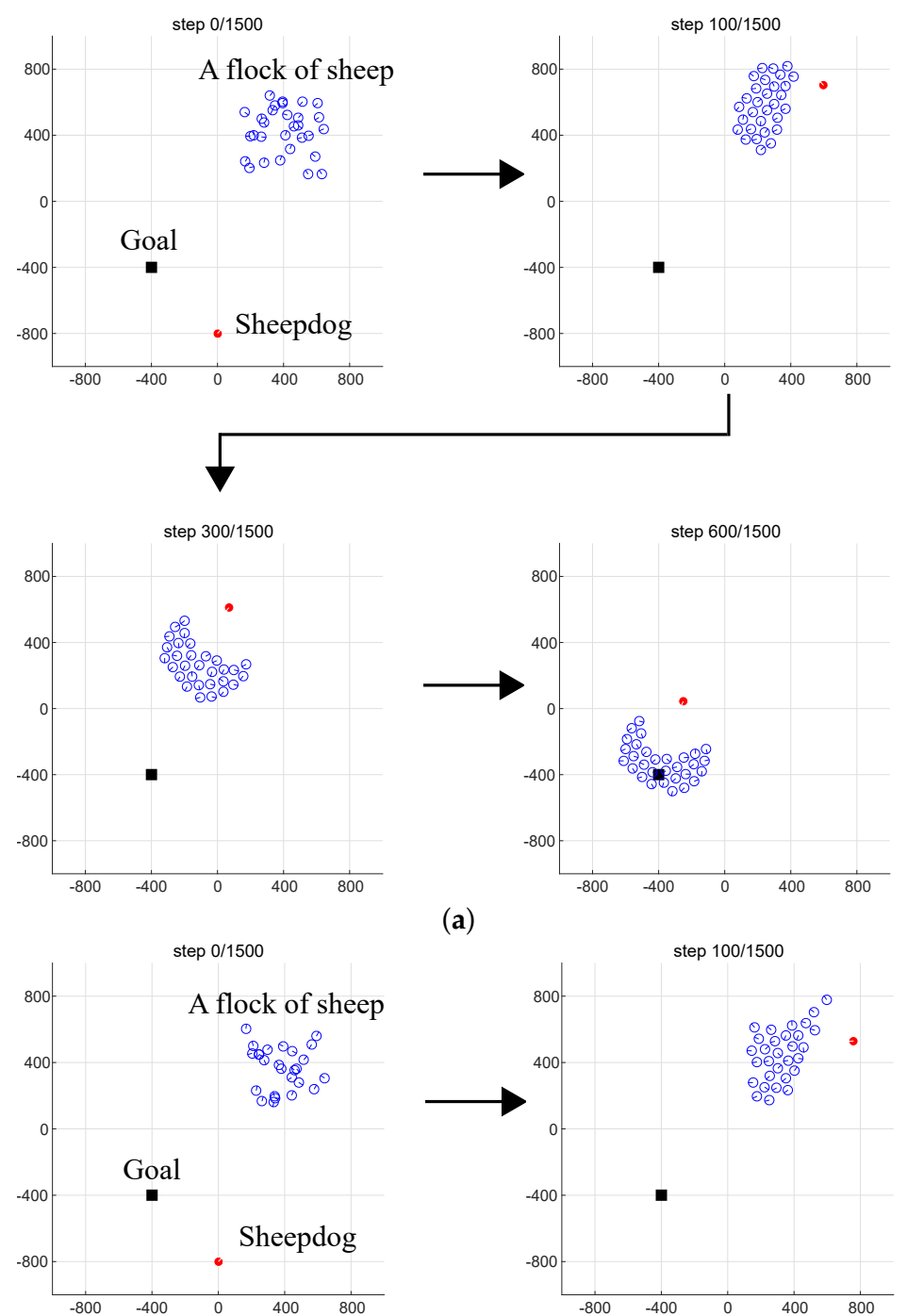

(a) $\quad$ step $100 / 1500$
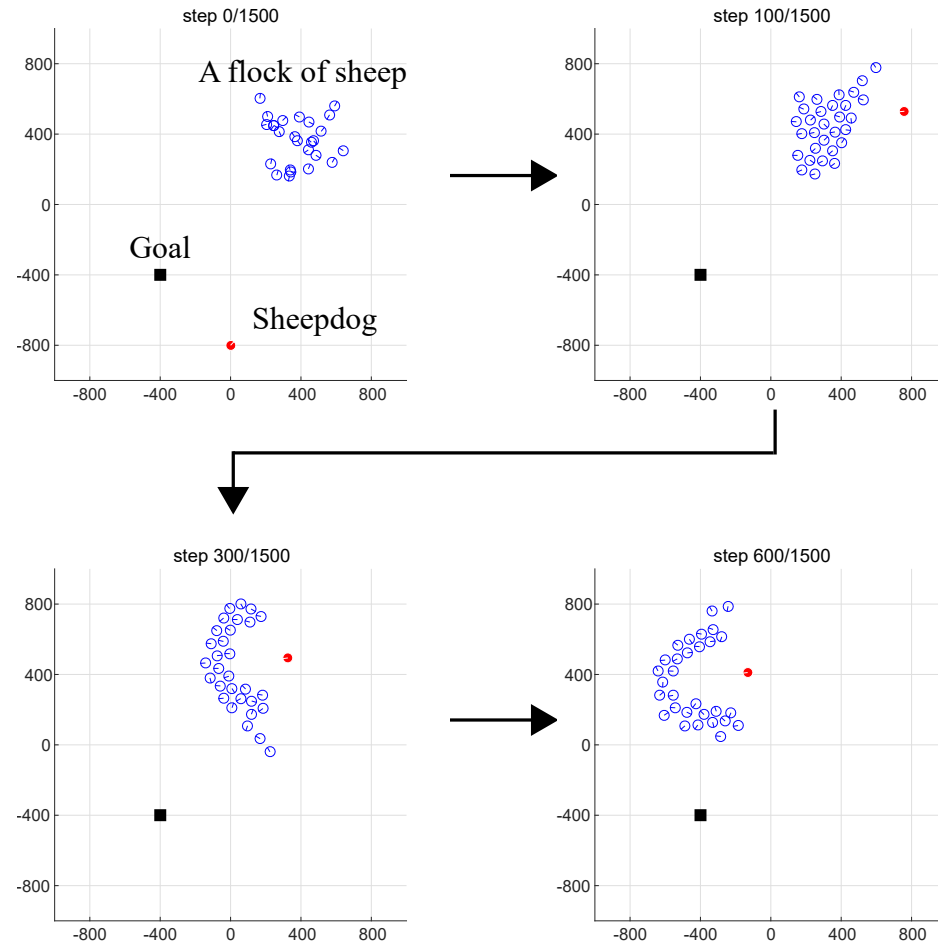

(b)

Figure 9. Effect of the size on navigation based on the center-targeting control. (a) Navigation successes on the condition that the sheep and the robot are treated as points; (b) Navigation failures on the condition that the sheep and the robot possess sizes. 


\subsection{Proposed Method: Method That Supplements the Goal Position with Other Sheep}

This sub-section considers the question: In a case in which a sheepdog cannot perceive the goal, what kind of decision does this cause the sheepdog to make? In this paper, the idea, "Using another object that replaces the goal as a substitute goal" is proposed, and a method for receiving a certain repulsion from a specified sheep instead of the goal is proposed, as shown in Figure 10b. This method works as follows:

1. When the goal is visible, the positional relationship of the position of the goal to the center of mass of the sheep (visible zone) is remembered.

2. When the goal has become invisible, the relative relationship of the position of the goal just before it became invisible with the direction of the center of the sheep is compared. If the position of the goal is to the left of the center of the sheep, the goal is the sheep on the leftmost side of the sheep that are visible. The same rule applies in the case where it is to the right.

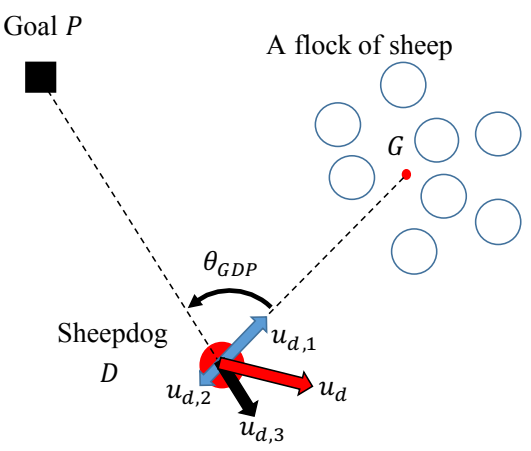

(a)

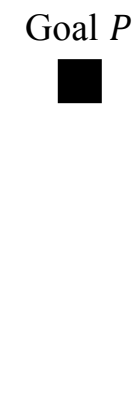

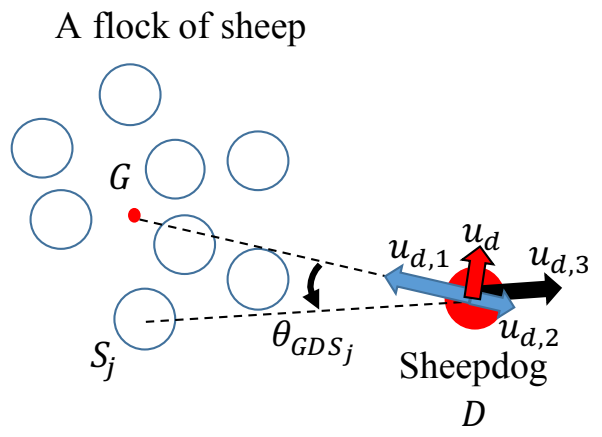

(b)

Figure 10. Dog controller: switching of its target between goal-visible cases and goal lost cases. (a) Design of the sheepdog controller for goal-visible cases; (b) Design of the sheepdog controller for goal-lost cases.

Specifically, the sheepdog sets the sheep that will receive repulsion as follows. First, just before the goal becomes invisible (Figure 10b), the unit vector to the goal from the sheepdog is $e_{P^{\prime}} \in \mathbb{R}^{2}$, and the unit vector from the sheepdog to the center of the flock of sheep is $e_{G^{\prime}} \in \mathbb{R}^{2}$. At this time, if the angle formed by $e_{G^{\prime}}$ and $e_{P^{\prime}}$ is $\theta_{G D P} \in \mathbb{R}$, the following formula is established.

$$
\theta_{G D P}=\arcsin \left(\left(e_{G^{\prime}} \times e_{P^{\prime}}\right)_{z}\right)
$$

Next, the unit vector from the sheepdog to the center of the flock of sheep under present conditions (Figure $10 \mathrm{~b}$ ) is $e_{G} \in \mathbb{R}^{2}$, and the unit vector from the sheepdog to the $i$ th sheep $S_{i}$ is $e_{S_{i}} \in \mathbb{R}^{2}$. Similar to Formula (17), if the angle formed by $e_{G}$ and $e_{S_{i}}$ is $\theta_{G D S_{i}} \in \mathbb{R}$, the following formula is established.

$$
\theta_{G D S_{i}}=\arcsin \left(\left(e_{G} \times e_{S_{i}}\right)_{z}\right)
$$

So, it is assumed that the sheepdog receives repulsion from the sheep $S_{j}(j=1,2, \cdots, n)$ with the highest $\theta_{G D P} \theta_{G D S_{i}}$. This means that when $\theta_{G D P}>0$, by making this decision, in a case where the goal is to the left of the center of the flock from the perspective of the sheepdog, the sheep for which the angle is furthest to the left of the center of the flock receives repulsion, and similarly when $\theta_{G D P}<0$, in a case where the goal is to the right of the center of the flock, the sheep for which the angle is furthest to the right of the center of the flock receives repulsion. Additionally, in the case where the flock of sheep has sufficiently compacted, the instant when the goal became invisible to the sheepdog, $\theta_{G D P} \simeq \theta_{G D S_{j}}$. 
Because it is assumed that force is not received from an agent that cannot be perceived, the sheepdog calculates the center of the flock of sheep that it can perceive and calculates the movement vector as in Formulas (21) and (22). With the proposed method, the sheep that is the substitute for the goal is selected from among sheep that the sheepdog can perceive. The speed $u_{d}[k]$ and the position $x_{d}[k+1]$ of the sheepdog in the invisible goal case are calculated as shown below.

$$
\begin{aligned}
u_{d, 1}[k] & =\frac{x_{g}[k]-x_{d}[k]}{\left|x_{g}[k]-x_{d}[k]\right|} \\
u_{d, 2}[k] & =-\frac{x_{g}[k]-x_{d}[k]}{\left|x_{g}[k]-x_{d}[k]\right|^{3}} \\
u_{d, 3}[k] & =-\frac{x_{j}[k]-x_{d}[k]}{\left|x_{j}[k]-x_{d}[k]\right|} \\
j & =\underset{i}{\arg \max \left(\theta_{G D P} \theta_{G D S_{i}}\right)} \\
u_{d}[k] & =K_{d_{1}} u_{d, 1}[k]+K_{d_{2}} u_{d, 2}[k]+K_{d_{3}} u_{d, 3}[k] \\
x_{d}[k+1] & =x_{d}[k]+u_{d}[k]
\end{aligned}
$$

\subsection{Analysis of the Impact of the Proposed Method on Guidance}

To quantify guidance performance, the following indices are introduced.

- Guidance distance: $d$ : the value when the distance between the center of the flock of sheep and the goal is lowest during guidance is defined as the guidance distance.

Guidance distance $d \in \mathbb{R}_{+}$can be represented by the following formula assuming that the position of the center of the sheep is $x_{g}[k]$, and the position of the goal is $x_{p}$.

$$
d=\min _{k}\left|x_{g}[k]-x_{p}\right|
$$

Figure 11 shows a view of guidance based on the proposed method. In Figure 11, the sheep that receives repulsion as a substitute when the sheepdog cannot perceive the goal is represented in blue. From Figure 11, it can be concluded that the proposed method functions well, with the key to successful guiding assumed to be cyclic movement from left and right by the sheepdog as shown in Figure 12. From the perspective of the sheepdog, the goal appears to be on the left side of the center of the sheep (Figure 12 (top left)). This changes to the situation in which the goal cannot be perceived and repulsion is received from the sheep on the left side (Figure 12 (top right)). Then the situation changes such that the goal is visible on the right (Figure 12 (lower right)), and then after passing through the situation in which the goal cannot be perceived and repulsion is received from the sheep on the right (Figure 12, (lower left)), the situation returns to one in which the goal is again visible on the left (Figure 12 (upper left). The sheepdog can guide the sheep towards the goal by repeating such a cycle of movements.

Next, as the number of sheep is varied from 1 to 50 , the guidance performance under the increase of the effect of blind zones was analyzed. The experiment was done 50 times for each flock size of sheep. The results are shown in Figure 13. The number of sheep is represented on the horizontal axis, and the average guided distance when the test was performed 50 times is represented on the vertical axis. Figure 13 reveals that using the previous method, as the size of the flock increases, the guided distance also increases. With the proposed method on the other hand, the guided distance does not change very much, revealing that stable guidance is possible as the number of sheep increases. It shows that when there are 30 sheep, using the previous method, the guided distance is about 600, but using the proposed method, it is about 50. Figure 14 visually represents the guided distances. When the situation where the guided distance is 700 (Figure 14a) and the situation where the guided 
distance is 100 (Figure 14b) are compared, it is confirmed that there is a great difference in the guidance performance of the previous method and the proposed method.
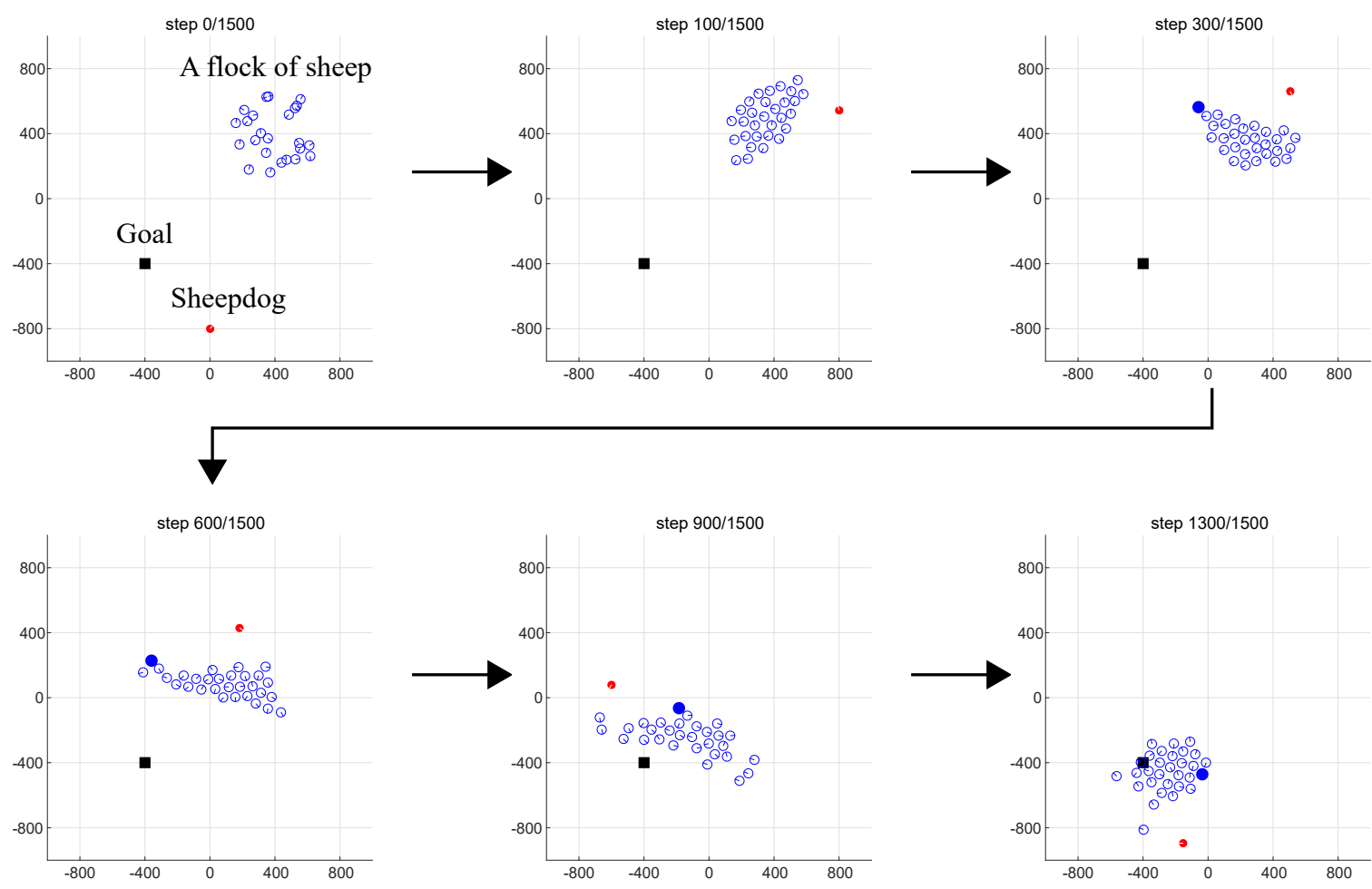

Figure 11. Snapshots of the sheep and the sheepdog position based on the proposed control method.

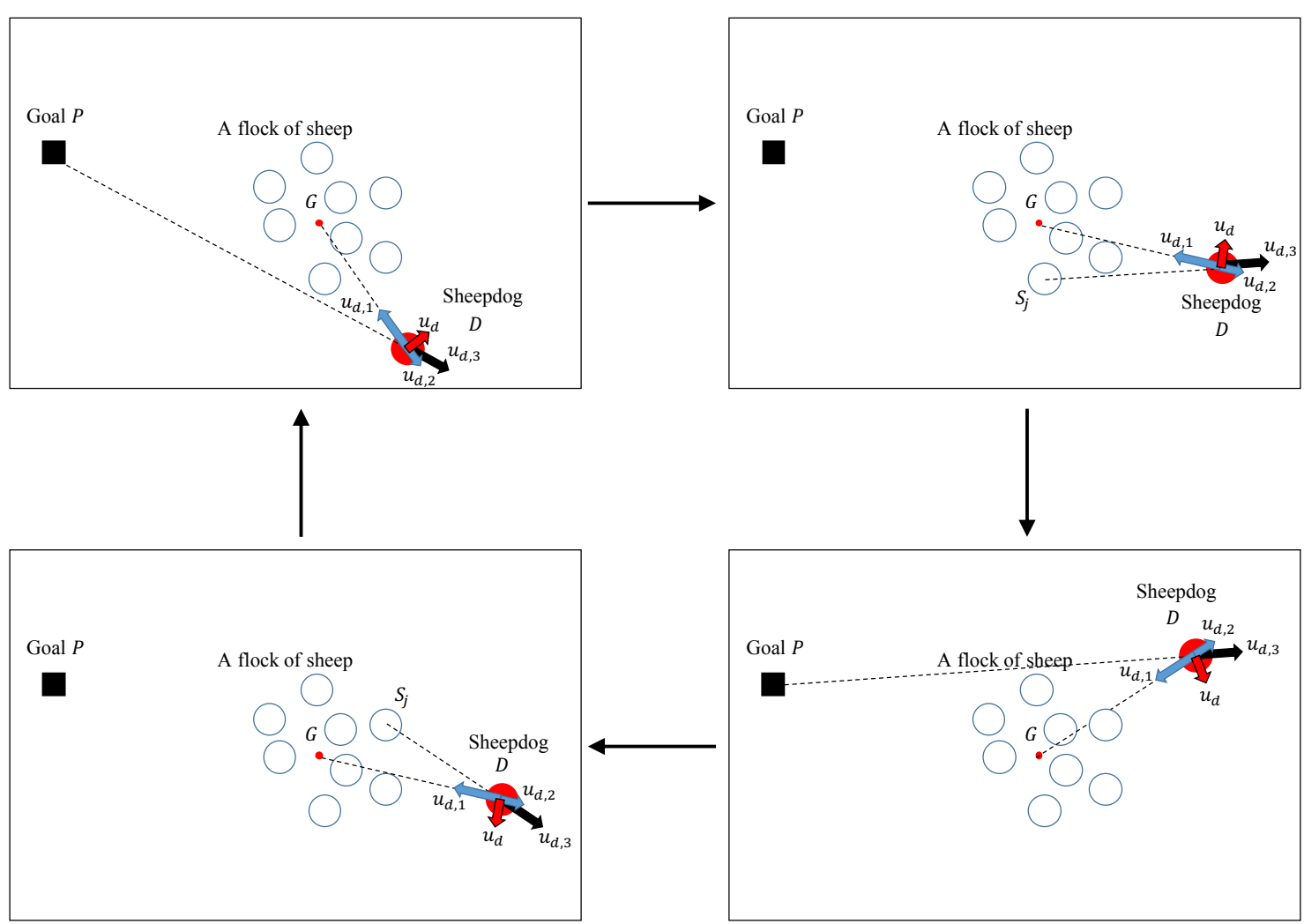

Figure 12. Guidance successes thanks to the periodic motions of the sheepdog for goal-lost-situation. 


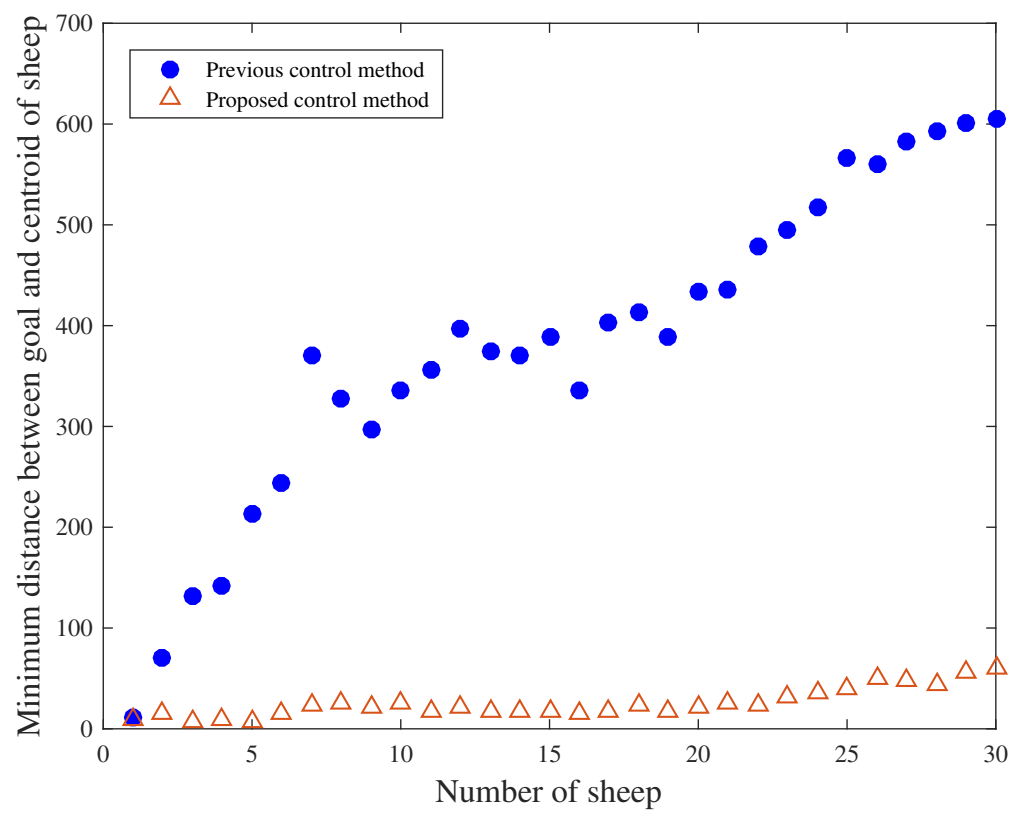

Figure 13. Relation between the number of sheep and the average of minimum guided distance.

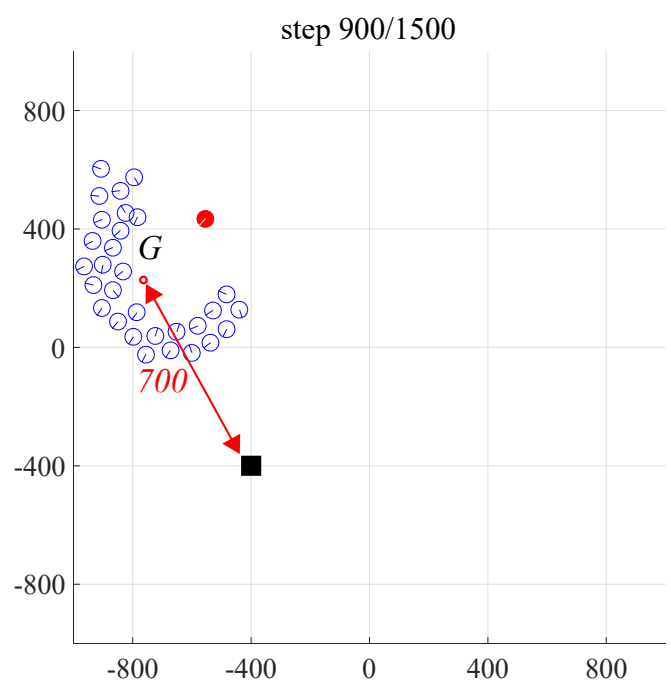

(a)

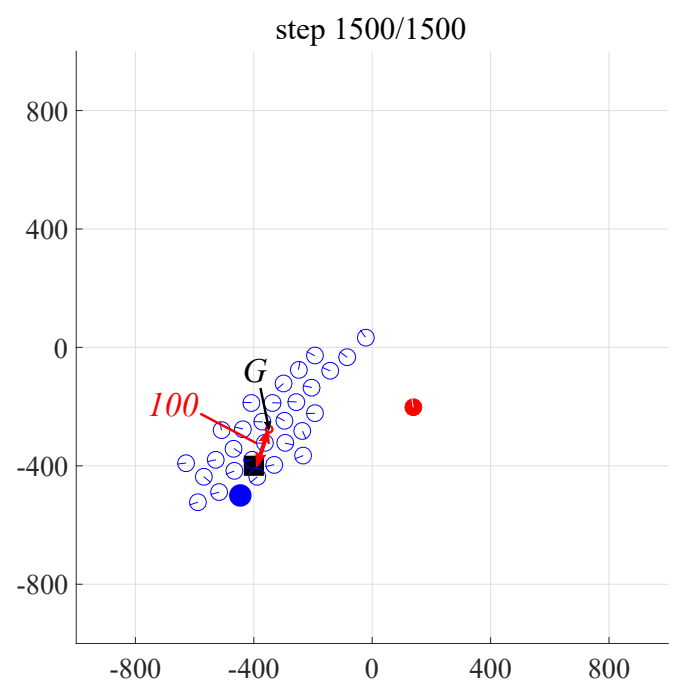

(b)

Figure 14. Success and failure cases. (a) Previous control method: $d=700$; (b) Proposed control method: $d=100$.

\section{Conclusions}

This paper tries to demonstrate the sheepdog-type navigation, as simply as possible, which is based on the concept of a sheepdog guiding a large flock of sheep. With reference to past research, a model for sheep and a model for a sheepdog were built, and then a real-world robot experiment was done. During the experiment, it was confirmed that guidance is possible in cases with a small number of sheep robots, and at the same time, it was noticed that an increase in the number of sheep was accompanied by the appearance of "objects that cannot be perceived." A simulation model was therefore built, and cases where there are dead zones (blind zones) were analyzed. It was confirmed that using the previous method, as the number of sheep increased, the goal position was obscured making it invisible to the sheepdog, resulting in guidance failure. It was also confirmed that in cases 
where the goal becomes invisible, selecting an appropriate object other than the goal and setting this object as the goal does resolve the problem and maintains the guiding performance even as the number of sheep increases. In the future, the effectiveness of the proposed method will be verified through real-world experiments using more sheep.

Author Contributions: Y.S., M.I. and K.O. developed the controller for goal-lost-situation, M.I. performed the experiments and analyzed the data, and Y.S., M.I. and K.O. wrote the paper.

Acknowledgments: This paper was supported by JST CREST Grant Number JPMJCR14D5, and JSPS KAKENHI Grant Number 18K13776, Japan.

Conflicts of Interest: The authors declare no conflict of interest.

\section{References}

1. Correll, N.; Schwager, M.; Rus, D. Social control of herd animals by integration of artificially controlled congeners. In Proceedings of the International Conference on the Simulation of Adaptive Behavior, Osaka, Japan, 7-12 July 2008; pp. 437-446.

2. Butler, Z.; Corke, P.; Peterson, R.; Rus, D. From robots to animals: Virtual fences for controlling cattle. Int. J. Robot. Res. 2006, 25, 485-508. [CrossRef]

3. Juang, P.; Oki, H.; Wang, Y.; Martonosi, M.; Peh, L.S.; Rubenstein, D. Energy efficient computing for wildlife tracking: Design tradeoffs and early experiences with zebranet. In Proceedings of the 10th International Conference on Architectural Support for Programming Languages and Operating Systems (ASPLOS X), San Jose, CA, USA, 5-9 October 2002; pp. 96-107.

4. Balch, T.; Khan, Z.; Veloso, M. Automatically tracking and analyzing the behavior of live insect colonies. In Proceedings of the Fifth International Conference on Autonomous Agents (AGENTS '01), Montreal, QC, Canada, 28 May 2001; pp. 521-528.

5. Colot, A.; Caprari, G.; Siegwart, R. Insbot: Design of an autonomous mini mobile robot able to interact with cockroaches. In Proceedings of the 2004 IEEE International Conference on Robotics and Automation (ICRA '04), New Orleans, LA, USA, 26 April-1 May 2004.

6. Balch, T.; Arkin, R.C. Behavior-based formation control for multiple mobile robots. IEEE Trans. Robot. Autom. 1998, 14, 929-939. [CrossRef]

7. Reif, J.H.; Wang, H. Social potential fields: A distributed behavioral control for autonomous robots. Robot. Auton. Syst. 1999, 27, 171-194. [CrossRef]

8. Vaughan, R.; Sumpter, N.; Frost, A.; Cameron, S. Robot sheepdog project achieves automatic flock control. In From Animals to Animats 5: Proceedings of the Fifth International Conference on Simulation of Adaptive Behavior; MIT Press: Cambridge, MA, USA, 1998; pp. 489-493.

9. Sueoka, Y.; Kita, T.; Ishikawa, M.; Osuka, K. Harnessing control of sheepdog agents by on-line clustering. In Proceedings of the International Symposium on Nonlinear Theory and its Applications (NOLTA), Santa Fe, NM, USA, 8-11 September 2013; pp. 49-52.

10. Azuma, S.I.; Tabuchi, A.; Sugie, T. Modeling of sheepdog control. Trans. Soc. Instrum. Control Eng. 2012, 48, 882-888. (In Japanese) [CrossRef]

11. Fujioka, K.; Hayashi, S. Effective shepherding behaviours using multi-agent systems. In Proceedings of the IEEE Region 10 Conference, Singapore, 22-25 November 2016; pp. 3179-3182.

12. Lien, J.M.; Bayazit, O.B.; Sowell, R.T.; Rodriguez, S.; Amato, N.M. Shepherding behaviors. In Proceedings of the IEEE International Conference on Robotics and Automation (ICRA '04), New Orleans, LA, USA, 26 April-1 May 2004; pp. 4159-4164.

13. Strömbom, D.; Mann, R.P.; Wilson, A.M.; Hailes, S.; Morton, A.J.; Sumpter, D.J.; King, A.J. Solving the shepherding problem: Heuristics for herding autonomous, interacting agents. J. R. Soc. Interface 2014, 11, 145-151. [CrossRef] [PubMed]

14. Bennett, B.; Trafankowski, M. A Comparative investigation of herding algorithms. In Proceedings of the Understanding and Modelling Collective Phenomena (UMoCoP), Birmingham, UK, 2-6 July 2012; pp. 33-38. 
15. Couzin, I.D.; Krause, J.; James, R.; Ruxton, G.D.; Franks, N.R. Collective memory and spatial sorting in animal groups. J. Theor. Biol. 2002, 218, 1-11. [CrossRef] [PubMed]

16. Reynolds, C.W. Flocks, herds and schools: A distributed behavioral model. In Proceedings of the ACM SIGGRAPH Computer Graphics, Dallas, TX, USA, 18-22 August 1987; Volume 21, pp. 25-34.

(C) 2018 by the authors. Licensee MDPI, Basel, Switzerland. This article is an open access article distributed under the terms and conditions of the Creative Commons Attribution (CC BY) license (http:/ / creativecommons.org/licenses/by/4.0/). 